\title{
Observaciones sobre la fidelidad de territorio y expectativa de vida del Azulito Altoandino Xenodacnis parina (Thraupidae)
}

\author{
Juan Manuel Aguilar Ullauri \\ Departamento de Posgrados, Universidad del Azuay, Av. 24 de Mayo 7-77 y Hernán Malo, Código postal: 981, Cuenca, Ecuador. \\ Correo electrónico: juanmaguilaru@yahoo.com \\ Editado por/Edited by: Esteban Guevara \\ Recibido/Received: 21 diciembre 2018 Aceptado/Accepted: 03 abril 2020 \\ Publicado en línea/Published online: 19 julio 2020
}

Observations on territory fidelity and life expectancy of the Tit-like Dacnis Xenodacnis parina (Thraupidae)

\begin{abstract}
Little is known about the life expectancy of Andean birds. The Tit-like Dacnis Xenodacnis parina, distributed in Ecuador and Peru, is an Andean specialist found in patches of Polylepis characterized by the presence of Gynoxys shrubs. Several individuals were color banded at Cajas National Park in 2010. Three banded birds were recorded at the same locality 7 years after being banded. This study provides the first information on territory fidelity, social behavior, and longevity for the species, briefly evaluating the impact of road-killings. This information might prove important to support conservation actions for X.parina.
\end{abstract}

Keywords: Andes, behavior, color banding, fidelity, longevity, territories, threats.

\section{Resumen}

Se conoce poco sobre la expectativa de vida de las aves andinas. El Azulito Altoandino Xenodacnis parina, distribuido en Ecuador y Perú, es un ave especialista de los Andes que se encuentra en parches de Polylepis caracterizados por la presencia de arbustos del género Gynoxys. Se anillaron varios individuos de X. parina en el Parque Nacional Cajas, en 2010, con anillos de colores. Tres individuos fueron registrados en la misma localidad 7 años después de haber sido anillados. Este trabajo brinda la primera información de fidelidad de territorio, comportamiento social y longevidad de esta especie, y brevemente evalúa la mortalidad causada por atropellamientos. Estos datos pueden apoyar acciones de conservación para X. parina.

Palabras clave: Amenazas, Andes, anillamiento, comportamiento, fidelidad, longevidad, territorios.

Los Andes tropicales son un área importante en diversidad y endemismo (Myers et al., 2000). En los altos Andes tropicales se encuentran los bosques de Polylepis (Rosaceae) que, si bien no tienen una alta diversidad de aves, son hábitat de especies raras y especialistas que usualmente tienen distribuciones fraccionadas y están amenazadas por fragmentación de hábitat (Fjeldså, 1993; Donald et al., 2010). Una de estas especies es el Azulito Altoandino Xenodacnis parina (Thraupidae), distribuido discontinuamente en Ecuador y Perú, entre 3000-4400 m s.n.m. Esta especie muestra marcada predilección por zonas con presencia de arbustos de Gynoxys (Asteraceae), en donde se alimenta de áfidos y néctar extrafloral que se genera en el envés de las hojas de esta planta (Vuilleumier, 1984; Fjeldså, 1992; Fjeldså, 1993; Aguilar \& Iñiguez, 2015).

En septiembre de 2010, Aguilar \& Iñiguez (2015) colocaron anillos de colores a 20 individuos de Xenodacnis parina (nueve hembras, ocho machos y tres juveniles) en Illincocha, dentro del Parque Nacional Cajas (-2,766, -79,216; 4000 m s.n.m.). Mediante observaciones individuales de las aves anilladas, estos autores establecieron los ámbitos de hogar de seis de estos individuos en 680-7243 $\mathrm{m}^{2}$, dependiendo de la abundancia de arbustos de 
Gynoxys. Desde entonces, varios de los individuos anillados fueron observados casualmente en la misma localidad luego de ser monitoreados.

En febrero y septiembre de 2017 se observaron en la misma localidad tres individuos (dos machos y una hembra) que fueron anillados como juveniles en 2010. La observación tuvo lugar a pocos metros de donde fueron anillados, y estos individuos estaban acompañados de otros sin anillos. Esto indica que los individuos observados tenían al menos 7 años.

Xenodacnis parina es usualmente abundante (Fjeldså, 1993). Es probable que forme grupos familiares territoriales para disminuir la agresión intra-específica y aumentar el éxito reproductivo dado por este comportamiento cooperativo (Beletsky \& Orians, 1989; Armstrong, 1995). Estas observaciones reflejan que Gynoxys provee suficientes recursos para que estos grupos familiares permanezcan en su territorio. Incluso se ha registrado a la especie con normalidad durante una caída inusual de nieve (O'Neill \& Parker, 1978), lo que refleja su especialización a este recurso aun cuando los requerimientos energéticos para la termorregulación son altos (Londoño et al., 2015).

Aguilar (2016) encontró que los machos de la población del Parque Nacional Cajas son más pesados (media = 19,56 g; $\mathrm{DS}=0,9 ; \mathrm{N}=8$ ) que las hembras (media = 16,88 g; $\mathrm{DS}=1,36 ; \mathrm{N}=9$ ). Algunas aves de similar tamaño, también con dimorfismo sexual en masa corporal, tienen una expectativa de vida de 4-16 años (Lentino et al.; 2003; Wasser \& Sherman, 2010), por lo que el registro de al menos 7 años de longevidad reportado en este trabajo se encuentra dentro de un rango esperado.

Conocer la longevidad de una especie es importante en estudios demográficos, y contribuye a entender la evolución de los patrones de senescencia (Bornschein et al., 2015). Se ha propuesto que la longevidad está relacionada a la masa corporal, la dieta y la sociabilidad de las especies (Wasser \& Sherman, 2010). También, el clima de las altas elevaciones al parecer influye en la expectativa de vida de una población (Wasser \& Sherman, 2010), debido a que induce una menor carga parasitaria y menos competidores (Finch 1998).

Por otra parte, el 10 de mayo de 2012 un macho adulto anillado en 2010 fue encontrado muerto en la carretera que cruza por el Parque Nacional Cajas, a $60 \mathrm{~m}$ de donde había sido registrado dos años antes. Xenodacnis parina es una de las especies con mayor mortalidad por colisiones con vehículos (Aguilar et al., 2019), por lo que esta carretera podría ser una fuente importante de mortalidad para poblaciones cercanas a la vía. Desde septiembre de 2017 no se han registrados individuos anillados en esta localidad. Aunque aún se conoce poco de su historia natural, las observaciones de tres individuos anillados en sus mismos territorios al menos 7 años después de ser anillados demuestran fidelidad territorial y de hábitat. Se espera que esta información contribuya para la conservación de la especie.

\section{AGRADECIMIENTOS}

Agradezco a Pedro Álvarez, Paul Molina y Xavier Iñiguez por su apoyo constante y sus comentarios; también a Andrea Nieto por su compañía en el campo. Información utilizada en este trabajo tuvo el permiso de investigación 001-2014-SGA-PNC-BD-FA-Aguilar. Esta investigación fue parcialmente financiada por la Universidad del Azuay.

\section{REFERENCIAS}

Aguilar, J. M., \& Iñiguez, X. (2015). Hábitos alimentarios de Xenodacnis (Xenodacnis parina) en los páramos del sur del Ecuador. Ornitología Neotropical, 26 (2), 211-217.

URL: http://journals.sfu.ca/ornneo/index.php/ornneo/article/view/29

Aguilar, J. M. (2016). Description and conservation status of a new subspecies of Xenodacnis parina (Aves: Thraupidae) from the Ecuadorian Andes (Tesis de Maestría). Pontificia Universidad Católica del Ecuador, Quito. URL: http://repositorio.puce.edu.ec/handle/22000/10378

Aguilar, J. M., Nieto, A., Espinoza, N., Loja, G., \& Tinoco, B. A. (2019). Assessing patterns of bird roadkills in a high Andean Ecuadorian national park. Studies on Neotropical Fauna and Environment, 54, 149-156. DOI: http://dx.doi.org/10.1080/01650521.2019.1649953 
Armstrong, D. P. (1995). Effects of familiarity on the outcome of translocations, II. A test using New Zealand Robins. Biological Conservation, 71, 281-288. DOI: https://doi.org/10.1016/0006-3207(94)00038-R

Beletsky, L. D., \& Orians, G. H. (1989). Familiar neighbors enhance breeding success in birds. Proceedings of the National Academy of Sciences, 86, 7933-7936. URL: https://www.ncbi.nlm.nih.gov/pmc/articles/PMC298186/pdf/pnas00287-0281.pdf

Bornschein, M. R., Pizo, M., Sobotka, D. D., Belmonte-Lopes, R., Golec, C., Machado-de-Souza, T., Pie, M. R., \& Reinert, B. L. (2015). Longevity records and signs of aging in Marsh Antwren Formicivora acutirostris (Thamnophilidae). Wilson Journal of Ornithology, 127(1), 98-102. DOI: https://doi.org/10.1676/14-074.1

Donald, P. F., Collar, N. J., Marsden, S. J., \& Pain, D. J. (2010). Facing extinction: the world's rarest birds and the race to save them. Londres, Reino Unido: Bloomsbury Publishing.

Finch, C. E. (1998). Variations in senescence and longevity include the possibility of negligible senescence. The Journals of Gerontology Series A: Biological Sciences and Medical Sciences, 53(4), B235-B239. DOI: https://doi.org/10.1093/gerona/53A.4.B235

Fjeldså, J. (1992). Biogeography of the birds of the Polylepis woodlands of the Andes. En H. Balslev \& J. L. Luteyn (Eds), Paramo: an Andean ecosystem under human influence (pp. 31-44). Londres, Reino Unido: Academic Press.

Fjeldså, J. (1993). The avifauna of the Polylepis woodlands of the Andean highlands: the efficiency of basing conservation priorities on patterns of endemism. Bird Conservation International, 3, 37-55. DOI: https://doi.org/10.1017/S0959270900000770

Lentino, M, Bonaccorso, E., García, M. A., Fernández, E. A., Rivero, R., \& Portas, C. (2003). Longevity records of wild birds in the Henri Pittier National Park, Venezuela. Ornitología Neotropical, 14(4), 545-548. URL: https://sora.unm.edu/sites/default/files/journals/on/v014n04/p0545-p0548.pdf

Londoño, G. A., Chappell, M. A., Castañeda, M. D. R., Jankowski J. E., \& Robinson, S. K. (2015). Basal metabolism in tropical birds: latitude, altitude, and the 'pace of life'. Functional Ecology, 29(3), 338-346.DOI: https://doi.org/10.1111/1365-2435.12348

Myers, N., Mittermeier, R. A., Mittermeier, C. G., da Fonseca G. A. B., \& Kent, J. (2000). Biodiversity hotspots for conservation priorities. Nature, 403, 853-858. DOI: https://doi.org/10.1038/35002501

O'Neill, J. P., \& Parker III, T. A. (1978). Responses of birds to a snowstorm in the Andes of southern Peru. Wilson Bulletin, 90(3), 446-449. URL: https://sora.unm.edu/sites/default/files/journals/wilson/v090n03/p0446p0449.pdf

Vuilleumier, F. (1984). Patchy distribution and systematics of Oreomanes fraseri (Aves Coerebidae) of Andean Polylepis woodlands. American Museum Novitates, 2777, 1-17. URL: http://hdl.handle.net/2246/5260

Wasser, E., \& Sherman, W. (2010). Avian longevities and their interpretation under evolutionary theories of senescence. Journal of Zoology, 280, 103-155. DOI: https://doi.org/10.1111/j.1469-7998.2009.00671.x 